\title{
Fetal and maternal Doppler adaptation to maternal exercise during pregnancy: A randomized controlled trial
}

\author{
Irene Fernández Buihgas ${ }^{1}$, Aranzazu Martín Arias ${ }^{1}$, Marina Vargas-Terrones ${ }^{2}$, Maia Brik ${ }^{3}$, \\ Valeria Rolle ${ }^{4}$, Ruben Barakat ${ }^{2}$, María Muñoz Gonzalez ${ }^{1}$, Ignacio Refoyo ${ }^{2}$, Mar Gil Mira ${ }^{5}$, \\ and BELEN BSM SANTACRUZ MARTIN ${ }^{1}$
}

\author{
${ }^{1}$ Hospital Universitario de Torrejon \\ ${ }^{2}$ Universidad Politecnica de Madrid \\ ${ }^{3}$ Hospital Universitari Vall d'Hebron Servei de Ginecologia \\ ${ }^{4}$ Instituto de Investigación Sanitaria del Principado de Asturias \\ ${ }^{5}$ Universidad Francisco de Vitoria Facultad de Ciencias de la Salud
}

February 20, 2022

\begin{abstract}
Objective: To analyse the longitudinal effect of a supervised mild-moderate physical exercise program during pregnancy on uteroplacental and fetal Doppler. Design: Planned secondary analysis of an RCT Setting: Hospital Universitario de Torrejón, Madrid-Spain Sample: 124 women were randomized at 12+0-15+6 weeks to exercise vs non-exercise. Methods: Obstetric appointments were scheduled at about 12, 20, 28 and 36 weeks of gestation. GEE models were adjusted to assess longitudinal changes in Doppler measurements according to randomization group. Main Outcome Measures: Fetal middle cerebral artery, umbilical artery, cerebral placental ratio and uterine artery pulsatility index, were longitudinally measured by Doppler ultrasound throughout gestation, and normalized by MoMs or z-score calculation Results: No significant differences in fetal or maternal Doppler measurements were found at any check-up time-points. However, umbilical artery behaved differently in the exercise group where, starting from a not significantly higher pulsatility index, it showed a continuous decrease during pregnancy to a lower level than the non-exercise group, in which it remained unmodified throughout pregnancy. Conclusion: A regular supervised mild-moderate exercise program during pregnancy does not deteriorate fetal or maternal Doppler, suggesting that fetal wellbeing is not compromised. UAPI z-score decreases during pregnancy in the exercise group, while it remains stable in the non-exercise group. Funding statement: Research Grants were provided by Hospital Universitario de Torrejón (Madrid, Spain), Universidad Politécnica de Madrid (Madrid, Spain), Sanitas Healthcare and iMaterna Foundation.
\end{abstract}

\section{Hosted file}

Main document.docx available at https://authorea.com/users/461461/articles/557165-fetaland-maternal-doppler-adaptation-to-maternal-exercise-during-pregnancy-a-randomizedcontrolled-trial

\section{Hosted file}

Tables.docx available at https://authorea.com/users/461461/articles/557165-fetal-andmaternal-doppler-adaptation-to-maternal-exercise-during-pregnancy-a-randomizedcontrolled-trial

\section{Hosted file}

Figure 1.pptx available at https://authorea.com/users/461461/articles/557165-fetal- 
and-maternal-doppler-adaptation-to-maternal-exercise-during-pregnancy-a-randomizedcontrolled-trial

\section{Hosted file}

Figure 2.pptx available at https://authorea.com/users/461461/articles/557165-fetaland-maternal-doppler-adaptation-to-maternal-exercise-during-pregnancy-a-randomizedcontrolled-trial 